\title{
Numerical simulation of aerosols in an aircraft wake using a 3D LES solver and a detailed microphysical model
}

\author{
X. P. Vancassel ${ }^{1}$, Ph. Mirabel ${ }^{2}$, F. Garnier ${ }^{3}$ \\ [1] Onera - The French Aerospace Lab, F91761 Palaiseau Cedex, France \\ [2] Laboratoire des Matériaux, Surfaces et Procédés pour la Catalyse, Strasbourg, \\ France
}

[3] Ecole de Technologie Supérieure, Montréal, Canada

Correspondence to: F. Garnier (francois.garnier@etsmtl.ca)

Keywords: Aerosols, Aircraft Wake, Microphysics, Contrails, Large Eddy Simulation

\section{Abstract}

The global impact of aviation on the atmosphere is generally determined using the total amount of gas and particulate matter emitted at the aircraft engine exit but generally ignore some of the physical transformations occurring at much smaller scales in the aircraft wake. In that work, we present an offline alternative method based on the use of a detailed plume aerosol model combined to fluid trajectories calculated from 3D large-eddy simulations (LES). The study has been limited to the first $10 \mathrm{~s}$ behind a type Airbus 340 aircraft. The results have been compared to those obtained from a one-way coupling approach including a simple microphysics water vapor deposition model on soot cores. The respective evolutions of average ice particles radius are in good agreement. Furthermore, different types of aerosol properties are examined including the charged volatile particles, the dry and activated soot and the ice crystals from homogeneous and heterogeneous freezing. The variability of the aerosol size distribution clearly illustrates the influence of the mixing, as a function of the position in the aircraft plume. Finally, the volatile particles distribution exhibits a bimodal shape resulting from the presence of charges, in agreement with that observed in literature. 


\section{Introduction}

Emissions from aircraft engines include a large variety of gaseous and solid effluents (e.g. [1], [2]; [3]). Although these emissions are small compared to other anthropogenic surface emissions, (at the present time they represent about 2-3\% of all anthropogenic sources [4]), they are mostly released in the very sensitive region of the upper troposphere. In fact, aviation represents the only source of anthropogenic pollution at high altitudes, near and above the tropopause, where background concentrations of these trace substances are low and residence times long. The unique location of aircraft emissions and the predicted growth of air traffic demand require that particular attention is given to the potential effects of these emissions.

Perturbations of tropospheric chemistry by these effluents may contribute to human-induced climate change if they are large enough to modify the Earth's radiative budget balance, either directly by increasing greenhouse gases or indirectly by altering cloud formation processes at flight altitudes. Of particular importance are emissions of precursors of aerosols (ions, $\mathrm{SO}_{2}$, hydrocarbons) as well as precursors of contrails (soot, water vapor) which can lead to increased cloudiness in the form of persistent linear contrails and induced-cirrus clouds.

It is now well understood that these particles modify the atmosphere's properties at flight levels. Indeed, the emitted particles can influence the atmosphere's chemical composition by enhancing the background aerosol surface area density and the subsequent heterogeneous reactions that occur on the particles (e.g. [5], [6]). Contrail formation and possible cloud coverage increase also modify the Earth's radiative balance by absorbing and scattering light, and may contribute to climate change [4].

As contrails contribute significantly to aviation induced climate change and since air transport is expected to increase sharply, actions leading to a reduction of contrail formation are subject to numerous studies. It seems that, at the present time, one of the most promising ways to avoid contrail formation is to modify flight altitudes to avoid regions of high relative humidity. Other possibilities concern the use of alternative fuels and engine optimization.

However, a good evaluation of the efficiency of these processes requires a detailed knowledge of contrail and aerosols formation and evolution in the aircraft wake, as a 
function of flight and ambient conditions. However, despite large progress in this respect, considerable uncertainties remain about the formation processes, the evolution and long-term impact of particles in the upper atmosphere.

Contrail modelling in the near field of an aircraft engine is complex since it involves different multi-scale domains, such as fluid dynamics, chemistry, microphysics, and consequently is computationally very demanding for an accurate characterisation.

From the dynamics point of view, in the near field of the aircraft (less than 10 seconds after the emissions), the exhaust jets of the engine are entrained into wake vortices generated by the aircraft. The qualitative features of the engine jet/vortex interaction can be identified by two distinct phases. During the jet regime, the first few seconds after the emission, the jets rapidly mix with ambient air while the vorticity shed from the wings rolls up into a pair of trailing vortices. Afterward, in the wake regime the engine jets are entrained into the vortex flow and this phase extends downstream from 0.5 to about 10 wingspans ([7] and [8]). At this stage the action of these vortices causes a lateral stretching and a vertical compression of the engine jet and ultimately leads to quicker velocity decay, compared to the baseline co-flowing jet, generating large-scale fluctuations of the thermodynamics variables as well [9].

In the literature, most of the time trajectory box models are used to describe contrail formation and evolution. They are run along an average plume trajectory and ignore the large fluctuations in temperature and saturation ratio profiles. Since condensation phenomena are very sensitive to these profiles, the use of average trajectories can lead to large errors in the predictions. On the other hand very detailed aerosol and chemistry models have been used with simplified dynamics along the plume axis (or at the boundary), or using semi-empirical methods to calculate the plume mixing ([10], [11], [12], [13] and [14]).

In that paper, we present an offline alternative method based on the use of a detailed plume aerosol model associated to large-eddy simulation (LES) models for threedimensional simulations of the aircraft plume. In a first part, a series of LES calculations are compared by using online and offline microphysics modelling for ice particle formation and growth while a second part is focused on the study of the volatiles particles properties, especially size distribution, composition etc. A special 
emphasis will be put on the influence of mixing on new aerosols particle formation and their evolution in the near field of an aircraft wake.

The paper is then structured as follows: In Section 2.1, we start with a brief description of the LES code FLUDILES employed for the gas phase together with an ice growth model. Section 2.2 details the microphysics/aerosols models (ULP-ONE code) while the section 2.3 describes briefly the simulation setup. Section 3 compares the LES simulation results by using the online and the offline microphysics coupling for ice crystal growth. The advantages as well as the limitations of these two approaches are discussed. Finally, additional particles properties data as the size distribution and the composition for different types of aerosols are also analysed. The final section contains the conclusions of this work.

\section{Methodology and setup}

The work presented here is based on codes of fluid dynamics and microphysics operated online or coupled through an interface. Two microphysics schemes are considered: a simplified one for ice crystal growth which has been implemented online in the LES code while a more detailed one is used offline. So, the "online method" is based on a one-way interaction between the gaseous and the condensed phase through a simple ice condensation scheme, while in the "offline method" the gaseous and the condensed phases are treated sequentially leading to multiple interactions between the various types for aerosol particles. The different modelling configurations are presented in Figure 1 and are described in the next sections.

\subsection{FLUDILES: The LES code}

In FLUDILES, the gas phase is governed by the 3D compressible Navier-Stokes equations, together with a transport equation for the scalar field (i.e. water vapor). The dispersed phase is treated using a Lagrangian approach. As the description of FLUDILES has been largely presented in previous works (see for example [8], [15], and [16]), only the main equations are given below. 
In a Large-Eddy Simulation (LES) approach, any dimensionless variable $\phi(\mathrm{x})=\left[\rho_{\mathrm{g}}, \rho_{\mathrm{g}} \mathrm{u}_{\mathrm{i}}, \mathrm{E}_{\mathrm{g}}, \rho_{\mathrm{g}} \mathrm{Y}_{\mathrm{v}}\right]$ is then decomposed into a resolved part $\overline{\phi(\mathrm{x})}$, and a non-resolved (or subgrid-scale) part $\phi^{\prime \prime}(\mathrm{x})$ such as $\phi(\mathrm{x})=\overline{\phi(\mathrm{x})}+\phi^{\prime \prime}(\mathrm{x})$, where $\rho_{\mathrm{g}}$ represents the density of the gas constituted of the mixing air-water vapor, $u_{i}$ is the velocity, $E_{g}$ denotes the total energy, and $Y_{v}$ denotes the vapor mass ratio. For compressible flows, it is usual to introduce the Favre average [17] defined as $\tilde{\phi}=\overline{\rho_{\mathrm{g}} \phi} / \bar{\rho}_{\mathrm{g}}$. With this approach, the filtered conservation equations of a compressible gas yield:

$$
\frac{\partial \bar{\rho}_{\mathrm{g}}}{\partial \mathrm{t}}+\frac{\partial}{\partial \mathrm{x}_{\mathrm{i}}}\left(\bar{\rho}_{\mathrm{g}} \tilde{\mathrm{u}}_{\mathrm{i}}\right)=0
$$

$$
\frac{\partial}{\partial t}\left(\bar{\rho}_{\mathrm{g}} \tilde{\mathrm{u}}_{\mathrm{i}}\right)+\frac{\partial}{\partial \mathrm{x}_{\mathrm{j}}}\left(\bar{\rho}_{\mathrm{g}} \tilde{\mathrm{u}}_{\mathrm{i}} \tilde{\mathrm{u}}_{\mathrm{j}}\right)+\frac{\partial \overline{\mathrm{p}}_{\mathrm{g}}}{\partial \mathrm{x}_{\mathrm{i}}}-\frac{1}{\operatorname{Re}} \frac{\partial \tilde{\sigma}_{\mathrm{ij}}}{\partial \mathrm{x}_{\mathrm{j}}}=-\frac{\partial \tau_{\mathrm{ij}}}{\partial \mathrm{x}_{\mathrm{j}}}
$$

$$
\begin{aligned}
& \frac{\partial \overline{\mathrm{E}}_{\mathrm{g}}}{\partial \mathrm{t}}+\frac{\partial\left[\left(\overline{\mathrm{E}}_{\mathrm{g}}+\overline{\mathrm{p}}_{\mathrm{g}}\right) \tilde{\mathrm{u}}_{\mathrm{i}}\right]}{\partial \mathrm{x}_{\mathrm{i}}}-\frac{1}{\operatorname{Re}} \frac{\partial\left(\tilde{\mathrm{\sigma}}_{\mathrm{ij}} \tilde{\mathrm{u}}_{\mathrm{i}}\right)}{\partial \mathrm{x}_{\mathrm{i}}}-\frac{\partial}{\partial \mathrm{x}_{\mathrm{i}}}\left(\frac{\mu_{\mathrm{g}}\left(\tilde{\mathrm{T}}_{\mathrm{g}}\right)}{\operatorname{Re} \operatorname{Pr}(\gamma-1) \mathrm{M}^{2}} \frac{\partial \tilde{\mathrm{T}}_{\mathrm{g}}}{\partial \mathrm{x}_{\mathrm{i}}}\right)= \\
& \frac{\partial \mathrm{Q}_{\mathrm{i}}}{\partial \mathrm{x}_{\mathrm{i}}}-\frac{\partial \tau_{\mathrm{ij}} \tilde{\mathrm{u}}_{\mathrm{i}}}{\partial \mathrm{x}_{\mathrm{i}}}+\tau_{\mathrm{ij}} \frac{\partial \tilde{\mathrm{u}}_{\mathrm{i}}}{\partial \mathrm{x}_{\mathrm{i}}}+\frac{1}{\operatorname{Re}} \Theta
\end{aligned}
$$

$$
\frac{\partial}{\partial \mathrm{t}}\left(\bar{\rho}_{\mathrm{g}} \tilde{\mathrm{Y}}_{\mathrm{v}}\right)+\frac{\partial}{\partial \mathrm{x}_{\mathrm{i}}}\left(\bar{\rho}_{\mathrm{g}} \tilde{\mathrm{Y}}_{\mathrm{v}} \tilde{\mathrm{u}}_{\mathrm{i}}\right)-\frac{1}{\operatorname{Re} \operatorname{Pr}} \frac{\partial}{\partial \mathrm{x}_{\mathrm{i}}}\left(\mu_{\mathrm{g}}\left(\tilde{\mathrm{T}}_{\mathrm{g}}\right) \frac{\partial \tilde{\mathrm{Y}}_{\mathrm{v}}}{\partial \mathrm{x}_{\mathrm{i}}}\right)+\bar{\omega}=-\frac{\partial \xi_{\mathrm{i}}}{\partial \mathrm{x}_{\mathrm{i}}}
$$

Re and M are the Reynolds and Mach numbers, respectively. The Prandtl number Pr and the ratio of specific heats $\gamma=\mathrm{Cp} / \mathrm{Cv}$ are set equal to 0.7 and 1.4 , respectively, the admitted values for air. Temperature and pressure are related through the equation of state $P_{g}=\rho_{g} R_{g}$. 
In this description, the left-hand sides of Equations (1) to (4) are the transport equations expressed in terms of filtered variables, while the right-hand sides contain the so-called subgrid terms. These subgrid terms represent the effects of unresolved scales of motion on the large scales needing to be modelled, and are defined as follows:

The subgrid-scale stress (SGS) tensor, $\tau_{i j}=\bar{\rho}_{g}\left({\overline{u_{i}}}_{j}-\tilde{u}_{i} \tilde{u}_{j}\right)$, the SGS heat flux, $\mathrm{Q}_{\mathrm{i}}=\frac{1}{\gamma-1}\left(\overline{\mathrm{p}_{\mathrm{g}} \mathrm{u}_{\mathrm{i}}}-\overline{\mathrm{p}}_{\mathrm{g}} \tilde{\mathrm{u}}_{\mathrm{i}}\right)$ and the SGS vapor flux, $\xi_{\mathrm{i}}=\bar{\rho}_{\mathrm{g}}\left(\overline{\mathrm{Y}_{\mathrm{v}} \mathrm{u}_{\mathrm{i}}}-\tilde{\mathrm{Y}}_{\mathrm{v}} \tilde{\mathrm{u}}_{\mathrm{i}}\right)$.

We used the hybrid Smagorinsky model, which is the linear combination of an eddyviscosity-type model, the Smagorinsky model and the similarity model formulated by Liu-Meneveau-Katz [18]. It correlates well with the real subgrid scale stresses and adequately dissipates the energy in the small scales. It should be noted that the hybrid Smagorinsky model was successfully tested in the case of the jet/vortex interaction and experienced the best performances when dealing with turbulent stresses as well as the turbulent heat flux [19].

The source term $\bar{\omega}$ in the equation (4) for the mass vapor ratio is the rate of condensed matter, and is calculated using an ice particles growth equation expressed by a diffusion law as developed by Garnier et al., [16]. It assumes that all the soot particles emitted are hydrophilic and can therefore grow by water uptake. When the temperature decreases below a threshold, particles are allowed to freeze and then to grow to contrail-size ice crystals.

Note that the coupling terms which should appear in continuity, momentum and energy equations (Eqs. (2) to (4)) are assumed to be negligible, mainly due to the tracer limit and the low mass ratio between the solid (soot and ice particles) and the gas (air and water vapor) phases, which is of the order of a few percent. In addition, temperature cannot be modified by ice growth [3], and so thermal exchanges from particle to gas are also neglected. Finally, only mass exchange is considered through the term $\bar{\omega}$.

The equations (1) to (4) have been solved by using a sixth order compact scheme in space [20] and by a third order Runge-Kutta algorithm for time integration. A nonreflexive boundary condition has been used for the cross flow domain and periodic 
condition was employed in the axial direction so that temporal simulations can be done. We add a MSOU Superbee limiter for the transport equation for water vapor in Eq. (4).

As already mentioned, a Lagrangian particle tracking model has been used to represent the dispersed phase as an ensemble of particles moving along in the fluid (see [21] and [22] for details). The gas flow and particle equations are coupled at each time step of the computation. The position of the particle is calculated from the following equation using a fourth-order Adams-Bashforth method:

$$
\frac{d x_{\mathrm{p}}}{d t}=u_{\mathrm{p}}\left(x_{\mathrm{p}}, t\right)
$$

where $x_{p}$ and $u_{p}$ indicate position and velocity of particle $\mathrm{p}$, respectively. The particles dealt with here are fluid particles or numerical particles (i.e. an element of fluid containing physical particles). Their velocity and their thermodynamic properties (temperature, pressure, dilution, mixing ratios etc.) are evaluated locally [22]. This allows the reconstruction of an ensemble of fluid particles trajectories or "numerical particles", by recording the evolutions of $u_{\mathrm{p}}, T_{\mathrm{p}}$, and the dilution ratio which can be provided as input to any microphysical models.

The FLUDILES has been widely used for aircraft wakes and contrails related numerical studies in the past (e.g. [8], [9] and [16]).

\subsection{ULP-ONE code: Microphysics and aerosols interaction}

The second configuration is referred to as the "offline" method. As already mentioned, the model is based on a trajectory box model concept [23] or more recently [12] or [13] for instance). The exhaust plume is considered as a computational box moving along some trajectory calculated with the LES solver (i.e. FLUDILES) at each time step. Aerosol interaction calculations are carried out for the corresponding local variables such as temperature, and therefore the aerosols properties vary as a function of the considered location (i.e. as a function of the trajectory). This approach is explained in Figure 2. 
The volume change due to the entrainment of ambient air is taken into account from the temperature variation. Therefore, the plume overall dilution ratio $D$ is [11]:

$$
D(t)=\frac{T(t)-T_{\mathrm{a}}}{T_{0}-T_{\mathrm{a}}}
$$

where $T_{\mathrm{a}}$ is the ambient temperature and $T_{0}$ the plume temperature at the nozzle exit. Although the dilution ratio could have been obtained from a passive scalar evolution in the LES code, we have used, in a first attempt, the temperature change to determine the plume expansion, keeping in mind possible delay of the plume water supersaturation [12]. In this work, chemistry has not been taken into account. Therefore, the evolution of the mixing ratios of exhaust gases and particulate species is given by Kärcher [12]:

$$
\begin{aligned}
& \frac{d(\chi)}{d t}=\frac{d(\chi)}{d t}_{\text {mix }}+\frac{d(\chi)}{d t} \text { micro } \\
& \frac{d(\chi)}{d t}=\frac{d(\chi)}{d t}_{\text {nucl }}+\frac{d(\chi)}{d t}_{\text {cond }}+\frac{d(\chi)}{d t}_{\text {coag }}+\frac{d(\chi)}{d t} \text { frz }^{\frac{d}{d}}
\end{aligned}
$$

where the subscripts "mix" and "micro" stand for mixing and microphysics respectively, while "nuc", "cond", "coag" and "frz" stand for nucleation of volatile particles, condensation of water vapor and sulfuric acid, Brownian coagulation and heterogeneous freezing respectively.

The aircraft plume has been considered as a mixture composed of water vapor and sulphuric acid. This choice is justified by the amount of emitted water vapor (emission index $\mathrm{EI}_{\mathrm{H} 2 \mathrm{O}}=1.23 \mathrm{~kg} / \mathrm{kg}$ fuel burnt) and by the fact that sulphuric acid formed by oxidation of sulphur species included in the fuel, has a strong impact on the formation of new particles ([24], [25], [26], [27] and [28]). The fuel sulphur has been assumed to be sulphur dioxide ( $\mathrm{S}(\mathrm{IV})$ ). Its conversion into $\mathrm{S}(\mathrm{VI})\left(\mathrm{SO}_{3}\right.$ and $\left.\mathrm{H}_{2} \mathrm{SO}_{4}\right)$ has been assumed to be completed when dilution starts. The sulphur to sulphuric acid conversion factor was $2.5 \%$. Concerning aerosols, the classical heteromolecular homogeneous nucleation theory has not been used in our case although it is generally used to explain new particle formation in the atmosphere [29]. Yu and Turco [30] 
pointed out that the classical nucleation was not suitable for aircraft plumes as the sizes obtained were smaller than the observed. Several explanations can be proposed. First, very large quantities of chemi-ions have been detected in aircraft plumes [31], especially sulphuric acid ion clusters, and it has been shown that these clusters enhance particles formation and subsequent growth by collision mechanisms ([30], [14]). Second, during cooling of the plume, the use of a steady state is not adapted as the temperature change and therefore the thermodynamic properties variations of the plume are fast. Time-lag effects [32] are present and need to be corrected [33].

The use of a kinetic model is more appropriate in our case since it overcomes the difficulties mentioned above. Formation and growth of volatile particles is based on collision-aggregation processes between molecules, or between clusters or between molecules and clusters. The presence of electrical charges promotes particle growth since the coagulation kernel can be enhanced by a factor 100 (depending on the size of the colliding particles). The formation of sulphuric acid hydrates in the vapor phase is due to the strong binding energy, which exists between sulphuric acid and water molecules ([34] and [35]). Hydrates are initially distributed following the Wilemski and Wyslouzil self consistent assumptions [36] and ion cores are initialised separately at values of the order of $10^{17}$ ions/kg fuel [37]. Sensitivity studies by $\mathrm{Yu}$ and Turco [14] and Vancassel et al. [38] showed that engine exit concentration of chemi-ions can significantly modify the growth of volatile particles. The effect of inter particles forces, enhanced by electrical charges has been determined using Yu and Turco [14], from Fuchs [39].

As mentioned above, particle formation results from cluster collisions which are treated like coagulation of volatile particles:

$\frac{d N_{k}}{d t}=\frac{1}{2} \sum_{j=1}^{k-1} K_{k-j, j} N_{k-j} N_{j}-N_{k} \sum_{j=1}^{\infty} K_{k, j} N_{j}$

where $N_{\mathrm{k}}$ is the number density of particles containing k acid molecules (see [13] for a more detailed description). The non-iterative semi-implicit scheme developed by Jacobson et al. [40] for a stationary grid has been used as the coagulation algorithm. The coagulation kernels $K_{\mathrm{i}, \mathrm{j}}$ have been computed for each type of particle and electrostatic effects due to the presence of charges are also considered [13]. As most 
sulphuric acid molecules are hydrated, their interaction (condensation) with particles or clusters in the molecular regime has been treated as a special case of coagulation.

Condensation of water vapor onto volatile particles is calculated differently. Because of high dissymmetry between vapor pressures of sulphuric acid and water, it is assumed that during incorporation of sulphuric acid molecules, a cluster or particle is in quasi-equilibrium with respect to water [29]. Water vapor concentration on volatile particles is then adjusted at each time step, to its equilibrium value, using an iterative method. Changes in water vapor concentration determine whether water vapor condenses or evaporates from droplets.

Soot particles are taken into account in different forms. "Dry" soot particles are emitted by the combustor engine, with diameter in the range $20-60 \mathrm{~nm}$. The particle emission index used is of the order of $10^{15}$ particles $/ \mathrm{kg}$ fuel. This value probably represents an upper limit for modern engines' soot emissions (see for instance [28]). Soot particles emission indices found in the literature can vary by a factor 10 for the same aircraft but for different engines (e.g Airbus A340 CFM56 5C2/5C4 see [41], [28]).

Once sulphuric acid clusters and volatile particles form, they interact with soot particles, which may reach an activation criterion based on a soot particle's liquid mass fraction [42]. In the present study, soot particles are activated when approximately $10 \%$ of their total mass is composed of water-sulfuric acid mixture (see for instance Wong and Miake-Lye [43]). Activated soot particles are then allowed to grow by water uptake and may freeze if the required conditions are met, leading to contrail formation. The heterogeneous ice nucleation rate $J$ is worked out using the classical theory (see for example [44]), corrected for curvature effects [45] using an approximation of the nucleation rate per particle, per surface and time unit:

$$
J=\frac{N_{\mathrm{s}} k T}{h} \exp \left(-\frac{\Delta F_{\mathrm{g}}+\Delta F_{\text {act }}}{k T}\right)
$$

where $k$ is the Boltzmann constant, $h$ the Planck constant, $\Delta F_{g}$ the free energy of formation of the ice germ, $\Delta F_{\text {act }}$ the diffusion activation energy and $N_{s}$ the number of 
water monomers in contact with the ice surface. The critical germ radius is determined following Jensen et al. [46].

Soot scavenging by contrail particles and soot particles self-coagulation are not taken into account since the timescale for such processes (minutes to hours) is beyond the duration of the simulation (order of seconds). However, volatile particle collection by soot particles and ice particles is treated using the following coagulation equation:

$\frac{d N_{\mathrm{k}}}{d t}=-K_{\mathrm{ks}} N_{\mathrm{s}} N_{\mathrm{k}}$

which describes the rate of change of volatile particles concentration $N_{\mathrm{k}}$ due to coagulation with soot particles (concentration $N_{\mathrm{s}}$ ) at a coagulation rate $K_{\mathrm{ks}}$ [39].

Water uptake on activated soot particles (contrail particles) is determined by solving a transport equation, which is a function of the water vapor available in the plume and of the water (ice) saturation vapor pressure.

The particles are distributed over a size grid using a sectional approach (size binning). A quasi stationary structure is used so that the particle's volume is calculated exactly and interpolated each time step to the grid [47]. Soot particles are distributed as a function of the size of their inner core (carbonaceous material). The core-grid structure is fully stationary [47], so that the particles can grow by uptake of condensable material but the non volatile core does not get lost during evaporation. This approach is realistic as the initial dry soot distribution is conserved.

\subsection{Details of the computations}

Fluid dynamics of the near-field wake is investigated using temporal LES, as usually carried out in previous works (for example see details in [8], [9] and [15]). All the fluid reference variables are based on the core jet values, scaling the velocities with the centerline velocity of the jet $V_{j}$, and the characteristic length scale, equal to the jet radius R. For all the simulations completed, the Reynolds number, as function of the jet radius $\mathrm{R}=0.5 \mathrm{~m}$, is fixed to $\mathrm{Re}=\mathrm{V}_{\mathrm{j}} \mathrm{R} / \mathrm{v}=3.210^{6}$. The computational domain has the following dimensions: $\mathrm{Ly}=6 \mathrm{R}$ along the flight direction while in the cross plane $\mathrm{Lx}=\mathrm{Lz}=42 \mathrm{R}$ which is relatively large in order to avoid interaction with the 
boundaries. The mesh is uniform in the domain $-11.1 \mathrm{R}<\mathrm{x}, \mathrm{z}<11.1 \mathrm{R}$ with $\Delta \mathrm{x}=\Delta \mathrm{y}=\Delta \mathrm{z}=0.15 \mathrm{R}$ and stretched using a monotonic tanh law outside this domain.

The velocity field induced by a vortex wake is usually represented by a fully rolled-up Lamb-Ossen vortices with a core radius of $r_{c}=3 m$ and an initial circulation of $\Gamma_{0}=488.78 \mathrm{~m}^{2} \mathrm{~s}^{-1}$ for an Airbus 340-300.

In the Lagrangian approach, each particle is followed individually. Because the number density of soot particles is high we minimise the simulation cost by carrying on computational particles (of the order of 250,000 in the simulations), with each representing $\mathrm{N}$ physical particles. These latter particles are considered to be spherical, with a radius $r_{p}$ and they are treated as passive tracers due to their small size. Thus, they have the same velocity as the carrier phase and the drag force is then neglected. This is justified by the fact that the particle relaxation time remains small (of the order of $10^{-5} \mathrm{~s}$ for ice particles with the largest $r_{p}$ of a few microns) and negligible, as compared to the flow characteristic time [21].

\section{Results and discussion}

The results presented here have been obtained for an Airbus A340-300, flying at cruising altitude. The ambient conditions we have used are (pressure, temperature and relative humidity respectively) $240 \mathrm{hPa}, 220 \mathrm{~K}$ and $\mathrm{RH}=30 \%$. The aircraft velocity was $250 \mathrm{~m} \cdot \mathrm{s}^{-1}$ and the engine exit temperature was $580 \mathrm{~K}$. We have assumed that the fuel sulphur content (FSC) was 1000 ppm by mass, which is slightly above the average range of observed FSC. Under these conditions, the offline microphysical model (ULP-ONE) predicted contrail formation. The online code triggered ice crystal formation when the temperature reached $268 \mathrm{~K}$. Afterwards, the growth or evaporation of the particles was determined as a function of the water saturation vapor pressure above ice. For the offline case, the calculations have been done on 25,000 randomly chosen trajectories.

We have plotted in Figure 3 the average ice particle radius over these trajectories, as a function of time past behind the nozzle exit. In the online case, each element of fluid 
is associated to a numerical particle, containing $10^{6}$ identical physical particles, to limit the computing costs.

All the initial soot particles have the same radius of $20 \mathrm{~nm}$ (i.e. monodispersed distribution) and the physical particle population related to a numerical particle remains monodisperse. As ice particles form from soot and evolve (growth or evaporation), the associated radius of the numerical particles changes since different thermodynamic conditions are encountered in the aircraft wake. Consequently the aerosol population evolves into a polydisperse distribution. The mean radius is calculated as follows:

$r_{\text {mean }}^{\text {ONLINE }}(t)=\frac{1}{N_{\text {traj }}} \sum_{i=1}^{N_{\text {traj }}} r_{i}(t)$

Where $r_{\text {mean }}^{\text {ONLINE }}$ is the mean radius in the entire aircraft plume at time $t, N_{\text {traj }}$ the total number of selected trajectories (or numerical particles) and $r_{i}(t)$ is the radius of the particles in the $i$ element of fluid.

The offline case is slightly different as for each numerical particle, an aerosol population is determined and includes ice particles, "dry" or activated soot, volatile particles etc. Consequently, this population is then polydisperse. At the end of the run, in each numerical particle, a "volume equivalent" radius is determined for ice particles. Then, an average value is calculated for the ensemble of trajectories considered. It can be written as:

$$
r_{\text {mean }}^{\text {OFFLINE }}(t)=\frac{3}{4 \pi}\left(\frac{1}{N_{\text {traj }}} \sum_{i=1}^{\mathrm{N}_{\text {taj }}} \frac{\sum_{j=1}^{\mathrm{NBIN}}\left(\frac{4}{3} \pi r_{i, j, i c e}^{3}(t) n_{i, j, i c e}(t)\right)}{\sum_{j=1}^{\mathrm{NBIN}} n_{i, j, i c e}(t)}\right)^{1 / 3}
$$

where $r_{i, j, i c e}$ is the radius of an ice crystal in a size bin $j$ of the numerical particle $i$, $n_{i, j, i c e}$ is the ice particle concentration, NBIN the number of size bins.

The results plotted in Figure 3 show a good agreement between the offline method and the online method. The mean radius peaks at $0.65 \mu \mathrm{m}$ in both cases but the maximum is slightly delayed in the online case ( $0.6 \mathrm{~s}$ against $0.9 \mathrm{~s}$ approximately). 
The evolution is then very similar up to $4 \mathrm{~s}$ and from this point the gap increases. The offline method predicts a mean value of $0.190 \mu \mathrm{m}$ at $10 \mathrm{~s}$ against $0.225 \mu \mathrm{m}$ for the online case. The chosen atmospheric conditions were not supersaturated with respect to ice. Therefore, the contrail evaporates. The $35 \mathrm{~nm}$ difference is not really significant if we consider the respective approaches: different condensation scheme, different way of coupling processes and different thermodynamic data. To this respect, using different saturation vapor pressures for water can lead to discrepancies, especially concerning the calculation of the saturation ratio that determines the growth intensity. Besides, as mentioned in section 2 "methodology", the dilution ratio in the offline case has been determined from temperature variations, which can lead to errors in the saturation ratio calculations. We can also remember that variations can come from the contrail formation hypotheses used. In the offline method, it is based on the calculation of heterogeneous freezing on activated soot particles. Therefore, all the soot particles may not be activated nor frozen. When working out mean values, this fact can have an influence as the total amount of condensable material is distributed over the condensation sites. This can lead to the formation of fewer ice particles, but bigger in size or inversely.

But from this attempt, we can conclude that the agreement is remarkable for the determination of size properties mean values. Some refinements will be proposed in the conclusion section. Results in Figure 3 also show the evolution of the particle mean radius in the plume when using a parameterized overall dilution ratio as expressed by a simple formula based on plume measurements [41]:

$$
D(t)=\frac{A F R}{7000\left(t / t_{0}\right)^{0.8}}
$$

with $t_{0}=1 s$ as arbitrary reference scale and $A F R$ for air fuel ratio $(A F R=60)$. The curve exhibits a maximum value of $0.55 \mu \mathrm{m}$ very quickly after the engine exit. This value is slightly smaller than the two other cases maxima. Besides, the evolution after the peak is also quite different. Until 6 seconds in the plume, the slope is smoother than in the "online" and "offline" cases but the values drop very quickly after so that the size at 8 $\mathrm{s}$ is $0.1 \mu \mathrm{m}$ which is basically the size of activated soot particles (see for example the results from in situ observations performed by Schröder et al. [48]. This illustrates 
that different dilution calculations lead to significantly different mean sizes of ice crystals. In other words, using some dilution models may lead to the prediction that ice particles survive after $10 \mathrm{~s}$ when using detailed dilution calculations as some others (based mainly on parameterized dilution) may just predict that particles have evaporated before. This is of importance if we keep in mind that atmospheric larger scale models can be influenced by such results as they will be used as input data.

Some additional results are plotted in Figure 4. Two slices of the plume are shown at $\mathrm{t}=1.8 \mathrm{~s}$, for the offline and the online case. The values presented here are the ice particle mean radius for each numerical particle (i.e. for each position). As previously described, the "offline" radius is the average value of the ice particle worked out from the polydisperse size distribution at a given location. The "online" radius is the size of all the identical droplets at the same position.

Firstly, we can notice that the offline case shows a region at the centre of the roll-up plume where small values of ice particle size (dark blue in Figure 4) are detected. This indicates that at the vortex centre ice evaporates in this region, or that ice particles do not form or do not grow much. This is in contrast with the online method, where the ice particles are quite homogeneously distributed. As expected mixing has an influence on the particle properties, the position of small ice particles (or no ice particles) in the offline case is correlated to low temperature values and large dilution ratios. There is actually a competition between the influence of low temperatures which tend to promote condensation and growth and large plume dilution, which decreases water vapor concentration. In our results, although the same temperature fields are used for both online and offline cases, the two-dimensional slices do not illustrate the temperature effects in the same manner. Again, the fact we used the temperature as a dilution tracer can be invoked to explain the results obtained. Indeed, the effects of the transfer processes between the vapor and ice phases within the aircraft plume are only taken into account in the online method. Both the mass coupling and the thermal transfers are involved, through the rate of condensed matter $\varpi$, in the water vapor transport equation (Eq. 4) and the heat diffusion fluxes, which are proportional to the term $1 / \mathrm{M}^{2}$, as expressed in energy equation (Eq. 5), respectively. These transfers have a significant effect on the ice growth process and 
play then a role in the distribution of ice particle properties, as shown by Garnier et al., [16].

Figure 5 also shows the water vapor saturation ratio above ice in a two-dimensional slice of the plume at $\mathrm{t}=1.8 \mathrm{~s}$. The areas plotted in red indicate that there is supersaturation $(S>1)$ whereas the blue ones are subsaturated. The absence of ice particles or their small size is clearly correlated to the saturation ratio, as expected. The subsaturations at the centre and the tail of the plume are visible. The reasons previously invoked as well as the different thermodynamic data used can be responsible for the discrepancies pointed out here.

In Figure 6 is represented two-dimensional slice (Y-plane cut) of ice particle mean diameter, in the plume at 10 seconds behind the aircraft. At this stage, the results show that the ice particles are completely rolled-up around the vortex core. However, we can notice the presence of a ring of supersaturation, leading to sizes comprised between $0.6 \mu \mathrm{m}$ and $1.2 \mu \mathrm{m}$. As a consequence, in the vortex core, an inner zone of low saturation is observed where the growth by condensation is then extremely limited. Indeed, the fully turbulent jet is entrained by the vortex field and is completely wrapped around it. In the vortex core, due to the presence of a strong rigid-body-like flow [15], the mixing processes are clearly reduced leading to particles with the smallest diameters (less than $100 \mathrm{~nm}$ ).

One of the main interests about the offline method is also that the microphysical model used is far more detailed than the online one. It includes different types of aerosol populations such as the neutral volatile particles (non-electrically charged), the charged volatile particles (carrying one positive or negative electrical charge), the dry soot particles, the activated soot particles and the ice distribution from homogeneous or heterogeneous freezing. Besides, most of the relevant processes concerning aerosol interactions are taken into account. Therefore information about sulphate aerosol can be obtained; this is important as heterogeneous chemical reactions occur at the surface or inside particles in the atmosphere. Such reactions can be responsible for the activation of chlorine species for instance, which are involved in ozone destruction processes. They depend on the surface area density of the available aerosol and on their composition. The determination of aviation global 
impact is beyond the scope of this paper. However, we try to provide data to larger scale models users in order to better account for plume processes in CTM (Chemistry Transport Model) for atmospheric researchers.

The volatile particles evolution in the plume has also been monitored along the path of each numerical particle. One of the results (radius) is plotted in Figure 7. The volatile particle mean radius starts slightly above the sulphuric acid molecular size $(0.28 \mathrm{~nm})$. A huge number of hydrates are produced during the first few milliseconds after the engine and they may still be considered as gaseous clusters but as they participate to coagulation processes, they have been included in the average value calculations presented here. Therefore, the mean sizes are quite small. During the vortex roll-up, the average radius increases due to condensation processes and coagulation to values larger than $1.5 \mathrm{~nm}$. At $1.8 \mathrm{~s}$ particles reach sizes much larger than $3 \mathrm{~nm}$ in radius. Their composition is also determined (not plotted here). The data obtained depend on a large range of parameters; some of them being uncertain (like the fuel sulphur content, the sulphur conversion factor into sulphuric acid, the initial number of chemi ions). The size distribution is also available for the 25,000 computed numerical particles at any time of simulation. 50 of them at $3 \mathrm{~s}$ behind nozzle exit are plotted in Figure 8, along with the arithmetic mean value. This illustrates the variability of the size distribution, as a function of the position in the plume. The volatile particle distribution exhibits the bimodal shape showed by Yu and Turco [30] resulting from the presence of charges and from the use of variable accommodation coefficients for small particles [30].

We can notice that the first mode, made of clusters or very small particles (nm sized) can be inhibited, probably because of collection processes by bigger ice crystals [49]. The second size distribution plotted here concerns the activated soot particles. Contrarily to the online case, all the soot particles emitted do not from a contrail. Only some of them are activated, and their freezing rate is calculated as explained in section 2.2 (Eq. 8). Activated soot distribution peaks at $60 \mathrm{~nm}$, well beyond the mean diameter of dry soot, close to $40 \mathrm{~nm}$. The ice crystals distribution is finally plotted. The ice particles considered here come from heterogeneous freezing, i.e they are composed of an inner soot solid core, surrounded by ice. 
Although the time past the aircraft plume is small, such results can be used by mesoscale models whose size resolution can be increased so that vortex effects can be included. From this point, "Effective Emission Indices", taken into account alteration processes within the aircraft plume, can be determined, either for chemical species or for aerosols. Recent works indicate that using effective emission indices (EI) in these atmospheric models instead of engine exit EI lead to a significant difference in the aviation impact of $15 \%$ in the induced perturbation. This approach could be extended to the impact of particulate matter.

\section{Conclusion}

An extended trajectory box model strategy, based on Large-Eddy Simulations, has been developed to study new particle formation in aircraft exhaust plumes. This original method allows the use of detailed microphysics leading to multiple interactions between the various types of aerosol particles. The results have been compared to the results obtained from an online method. The spatial distribution of some of the particle properties points out discrepancies in the ice crystals mean size. Some areas are clearly subsaturated whereas some others remain supersaturated. Although the calculation strategies do not use the same approach, the difference in the trend observed can be connected to the coupling processes (gas/particles), different condensation scheme used, the thermodynamic data, the initialisation of the soot particle distribution etc. Nevertheless, the respective evolutions (in the offline and online case) of average values like the ice particles radius are in good agreement. To this respect, using the multi trajectory box approach is interesting as it also offers additional particles properties data like the size distribution, the composition for different types of aerosols such as the neutral and charged volatiles particles, the dry and activated soot particles and finally ice crystals formed from homogeneous or heterogeneous freezing. Firstly, the non-homogeneity of the particle size distribution as function of different locations within the aircraft wake is clearly shown and exhibits a bimodal shape resulting from the presence of charges. At the end of the simulations (10 s), the presence of a ring of supersaturation around the vortex core is 
highlighted leading to the biggest particles, and is associated to the existence of a zone adjacent to the vortex core, which promotes the condensation process.

This work still requires improvements, especially to take into account diffusion processes within the plume. But it is so far promising as it is meant to help reducing the uncertainties rising from plume effects in large scale models. Additional refinements can be done to improve the method; in particular including organic species would help in increasing the description of the volatile particles growth process. At the moment, organics have been included in a single box model. The interactions combinations from new aerosol types are numerous and increase the time of the run unacceptably. Finally one other further step would be to make proper comparisons with in situ measurements. 


\section{References}

[1] D.J. Hofmann, J.M. Rosen, Balloon observations of a particle layer injected by stratospheric aircraft at $23 \mathrm{~km}$, Geophys. Res. Let. 5, 511-514, 1978

[2] A. Petzold, J. Ström, F.P. Schröder, B. Kärcher, Carbonaceous aerosol in jet engine exhaust: emission characteristics and implications for heterogeneous chemical reactions, Atmos. Environ. 33, 2689-2698, 1999

[3] U. Schumann, On conditions for contrail formation from aircraft exhausts, Meteorol. Z. 5, 4-23, 1996

[4] Intergovernmental Panel on Climate Change (IPCC), 1999, Aviation and the Global Atmosphere, Penner J.E., Lister D.H., Griggs D.J., Dokken D.J., McFarland M. (Eds.), Cambridge Univ. Press, New-York, 1999

[5] B. Kärcher and S.K. Meilinger, Perturbation of the aerosol layer by aviation produced aerosols: a parameterization of plume processes. Geophys. Res. Let. 25, 4465-4468, 1998

[6] S.K. Meilinger, B. Kärcher, T. Peter, Microphysics and heterogeneous chemistry in aircraft plumes- High sensitivity on local meteorology and atmospheric composition. Atmos. Chem. Phys. 5, 533-545, 2005

[7] F. Garnier, C. Baudouin, N. Louinard, Engine Emission alteration in the nearfield of an aircraft. Atmos. Environ. 31, 1767-1781, 1997

[8] C. Ferreira-Gago, S. Brunet, F. Garnier, Numerical investigation of turbulent mixing in a jet/wake vortex interaction. AIAA J., 40: 276-284, 2002

[9] R. Paoli, F. Garnier, Interaction of exhausts jets and aircraft wake vortices: smallscale dynamics and potential microphysical-chemical transformations. C.R. Physique. $6,525-547,2005$

[10] R.C. Brown, R.C. Miake-Lye, M.R. Anderson, C.E. Kolb, T.J. Resch, Aerosol dynamics in near-field aircraft plumes, J. Geophys. Res. 101, 22939-22953, 1996

[11] G. Gleitsmann, R. Zellner, A modelling study of the formation of cloud condensation nuclei in the jet regime of aircraft plumes, J. Geophys. Res. 103, 1954319555,1998 
[12] B. Kärcher, A trajectory box model for aircraft exhaust plumes. J. Geophys. Res. $100,18835-18844,1995$

[13] A. Sorokin, X. Vancassel, P. Mirabel, On volatile particle formation in aircraft exhaust plumes, Phys. Chem. Earth (C) 26, 557-561, 2001

[14] F. Yu, R. P. Turco, The formation and evolution of aerosols in stratospheric aircraft plumes: Numerical simulations and comparisons with observations. J. Geophys. Res. 103, 25915-2593, 1998

[15] O. Labbé, E. Maglaras, F. Garnier, Large-eddy simulation of a turbulent jet and wake vortex interaction, Comp. Fluids,36, 772-785, 2007

[16] F. Garnier, E. Maglaras, F. Morency, X. Vancassel, Effect of compressibility on contrail ice particle growth in an engine jet, Int. J. Turbo Jet-Engines, 2013

[17] A. Favre, Équations des gaz turbulents compressibles, Journal de Mécanique, 4, pp. 361-390, 1965

[18] S. Liu, C. Meneveau, J. Katz, On the properties of similarity subgrid-scale models as deduced from measurements in a turbulent jet, J. Fluid Mech., 275, 83-119, 1994

[19] C. Ferreira Gago, F. Garnier, F. Utheza, Direct testing of subgrid scale models in large-eddy simulation of a non-isothermal turbulent jet, Int. J. Num. Meth. Fluids, 42, 999-1026, 2003

[20] S.K. Lele, Compact finite difference schemes with spectral-like resolution, J. Comp. Physics., 103, 16-42, 1992

[21] R. Paoli, J. Hélie, T. Poinsot, Contrails formation in aircraft wake. J. Fluid Mech. $502,361-373,2004$

[22] R. Paoli, X. Vancassel, F. Garnier, P. Mirabel Large-eddy simulation of a turbulent jet and a vortex sheet interaction: particle formation and evolution in the near field of an aircraft wake. Meteorol. Z. 17, 131-144, 2008

[23] CIAP Monograph 3: The stratosphere perturbed by propulsion effluents final report-DOT-TST-75-53, Department of transportation, Washington, 1975 
[24] C.A. Brock, F. Schröder, B. Kärcher, A. Petzold, R. Busen, M. Fiebig, Ultrafine particle size distributions measured in aircraft exhaust plumes. J. Geophys. Res. 105 26555-26567, 2000

[25] J. Curtius, B. Siereau, F. Arnold, R. Baumann, R. Busen, P. Schulte, U. Schumann, First direct sulfuric acid detection in the exhaust plume of a jet aircraft in flight, Geophys. Res. Let. 25, 923-926, 1998

[26] F. Schröder, C.A. Brock, R. Baumann, A. Petzold, R. Busen, P. Schulte, M. Fiebig, In situ studies on volatile jet exhaust particle emissions: Impact of fuel sulfur content and environmental conditions on nuclei mode aerosols, J. Geophys. Res. 105, 19941-19954, 2000

[27] U. Schumann, F. Arnold, R. Busen, J. Curtius, B. Kärcher, A. Kiendler, A. Petzold, H. Schlager, F. Schröder, K.-H. Wohlfrom. Influence of fuel sulfur on the composition of aircraft exhaust plumes: The experiments SULFUR 1-7, J. Geophys. Res. 107, 2002

[28] D.W. Fahey, E.R. Keim, K.A. Boering, C.A. Brock, J.C. Wilson, H.H. Jonsson, S. Anthony, T.F. Hanisco, P.O. Wennberg, R.C. Miake-Lye, R.J. Salawitch, N. Louisnard, E.L. Woodbridge, R.S. Gao, S.G. Donnelly, R.C. Wamsley, L.A. Del Negro, S. Solomon, B.C. Daube, S.C. Wofsy, C.R. Webster, R.D. May, K.K. Kelly, M. Loewenstein, J.R. Podolske, K.R. Chan, Emission measurements of the Concorde supersonic aircraft in the lower stratosphere, Science 270, 70-74, 1995

[29] P. Mirabel, J.L. Katz, Binary homogeneous nucleation as a mechanism for the formation of aerosols, J. Chem. Phys. 60, 3, 1138-1144, 1974

[30] F. Yu, R.P. Turco, The role of ions in the formation and evolution of particles in aircraft plumes, Geophys. Res. Let. 24, 1927-1930, 1997

[31] A. Frenzel, F. Arnold, Sulfuric acid cluster ion formation by jet engines: Implications for sulfuric acid formation and nucleation, DLR-Mitt. 94-06, Deutsches Zentrum für Luft und Raumfahrt, Köln, Allemagne, 106-112, 1994.

[32] B.E. Wyslouzil, G.J. Wilemski, Binary nucleation kinetics. III. Transient behaviour and time lags. J. Chem. Phys. 105, 1090-1100, 1996 
[33] A. Sorokin, X. Vancassel, P. Mirabel, Kinetic model for binary homogeneous nucleation in the $\mathrm{H}_{2} \mathrm{O}-\mathrm{H}_{2} \mathrm{SO}_{4}$ system: Comparison with experiments and classical theory of nucleation. J. Chem. Phys. 123, 244-508, 2005

[34] A. Jaecker-Voirol, P. Mirabel P., H. Reiss, Hydrates in supersaturated binary sulfuric acid-water vapor: a reexamination, J. Chem. Phys., 87, 4849-4852, 1987

[35] Noppel, M., H. Vehkamäki, M. Kulmala, An improved model for hydrate formation in sulfuric acid-water nucleation. J. Chem. Phys. 111, 218-228, 2002

[36] G. Wilemski, B.E. Wyslouzil, Binary nucleation kinetics. I. Self-consistent size distribution, J.Chem.Phys., 103, 3, 1127-1136, 1995

[37] F. Arnold, A. Kiendler, V. Wiedemer, S. Aberle, T. Stilp, Chemiion concentration measurements in jet engine exhaust at the ground: Implications for ion chemistry and aerosol formation in the wake of a jet aircraft, Geophys. Res. Let. 27 $1723-1726,2000$

[38] X. Vancassel, A. Sorokin, P. Mirabel, A. Petzold, C. Wilson, Volatile particles formation during PartEmis: a modelling study. Atmos. Chem. Phys. 4, 439-447, 2004

[39] N.A. Fuchs, The Mechanics of Aerosols, Pergamon Press, New York, 1964

[40] M.Z. Jacobson, R.P. Turco, E.J. Jensen, O.B. Toon, Modeling coagulation among particles of different composition and size, Atmos. Environ. 28, 1327-1337, 1994

[41] U. Schumann, H. Schlager, F. Arnold, R. Baumann, P. Haschberger, O. Klemm, Dilution of aircraft exhaust plumes at cruise altitudes, Atmos. Environ. 32, pp. 30973103, 1998

[42] C. Andronache, W.L. Chameides, Interactions between sulphur and soot emissions from aircraft and their role in contrail formation 1. Nucleation, J. Geophys. Res. 102, 21443-21451. 1997

[43] H.-W. Wong, R. C. Miake-Lye, Parametric studies of contrail ice particle formation in jet regime using one-dimensional microphysical modelling, Atmos. Chem. Phys. Discuss., 9, 22337-22363, 2010 
[44] H.R. Pruppacher, J.D. Klett, Microphysics of clouds and precipitation, $2^{\text {nd }}$ Ed., Kluwer Academic Publishers, 1997.

[45] N.H. Fletcher, Size effect in heterogeneous nucleation, J. Chem. Phys., 29, 572576,1958

[46] E.J. Jensen, O.B. Toon, S. Kinne, G.W. Sachse, B.E. Anderson, K. Roland Chan, C.H. Twohy, B. Gandrud, A. Heymsfield, R.C. Miake-Lye, Environmental conditions required for contrail formation and persistence, J. Geophys. Res. 103, 3929-3936, 1998

[47] M.Z. Jacobson, Fundamentals of atmospheric modelling, University Press, Cambridge, 2005

[48] Schröder F.P., Kärcher B., Petzold A., Baumann R., Busen R., Hoell C., Schumann U., Ultrafine aerosol particles in aircraft plumes: In situ observations. Geophys. Res. Let., 25, 2789-2792, 1998

[49] B. Kärcher, R. Busen, A. Petzold, F.P. Schröder, U. Schumann, J. Geophys. Res. 103, 17129-17147, 1998 


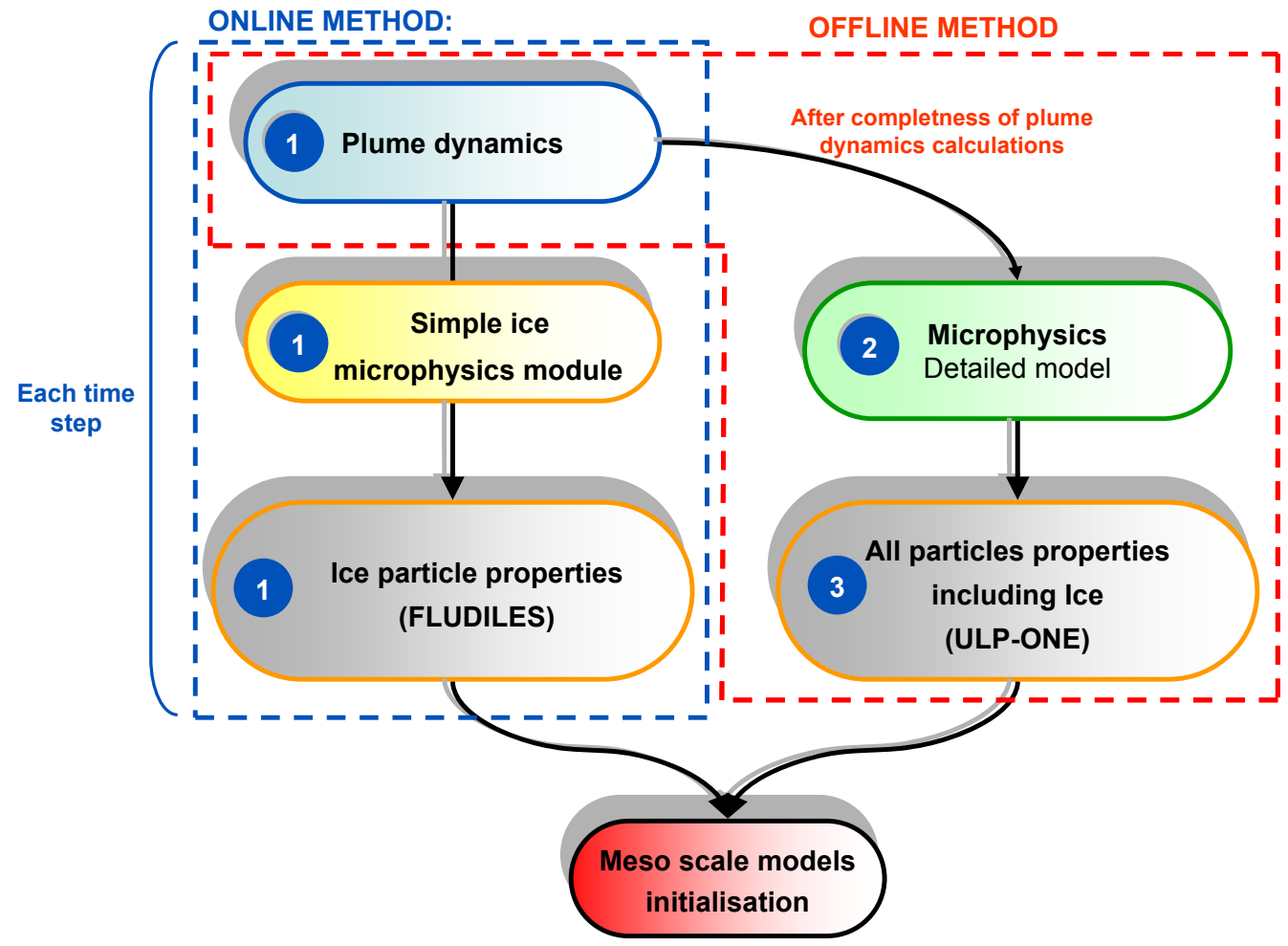

Figure 1: Flowchart to calculate the aerosol distribution in the plume with respect to the 2 configurations used in this work. The offline method is based on aerodynamics and detailed microphysics calculations carried out sequentially (1: plume dynamics calculations; 2: Microphysics calculations based on the established aerodynamics worked out in 1; 3: All properties determined for a plume slice. The online method uses a simple ice condensation scheme to treat contrail formation. The condensed phase equations are coupled to the fluid equations and solved simultaneously. 


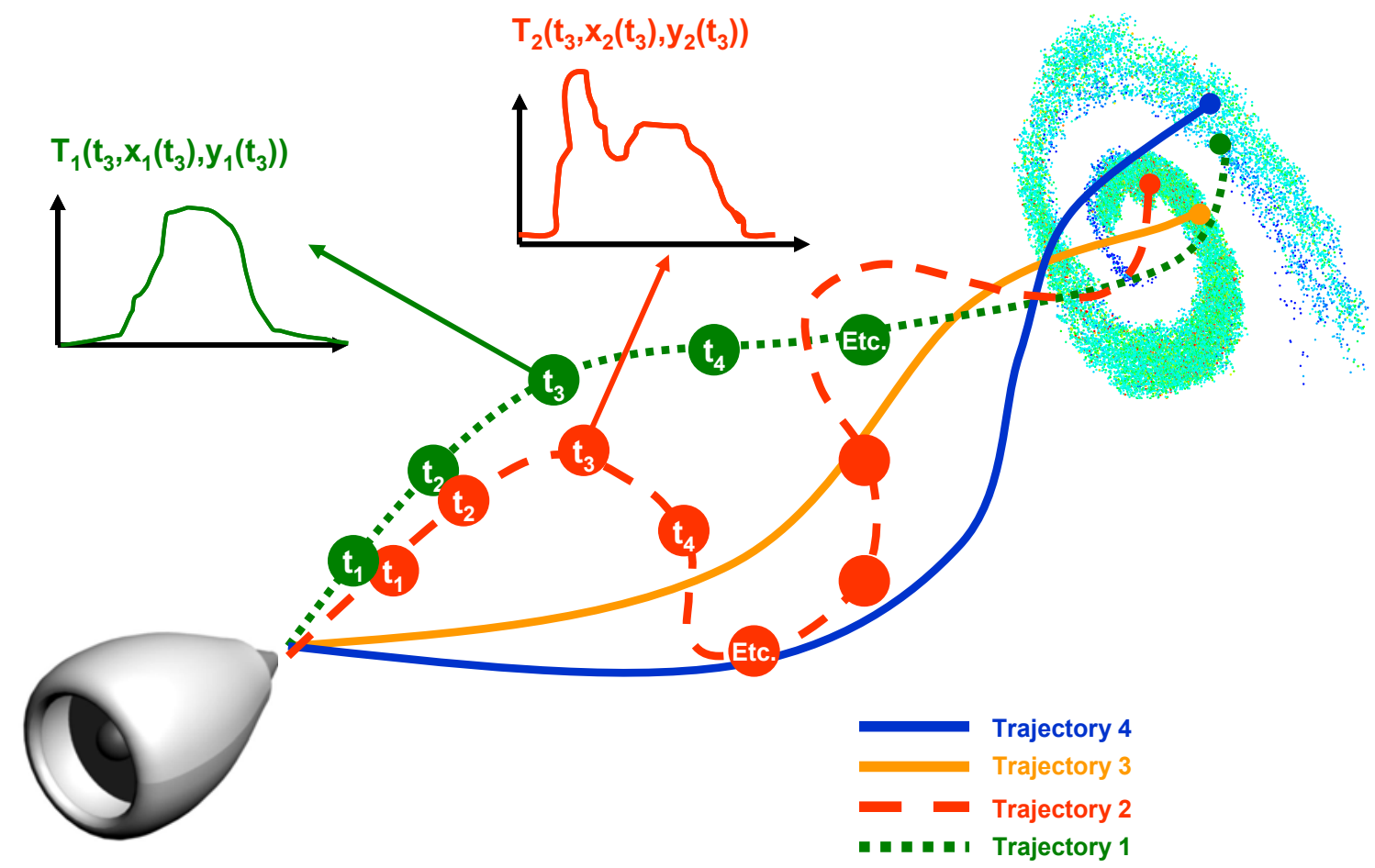

Figure 2: Diagram of the concept used for offline calculations. The trajectories are provided by FLUDILES from the nozzle exit to $10 \mathrm{~s}$ behind the aircraft. The microphysical model ULP-ONE is run for each fluid parcel with a regular time step $\left(t_{1}, t_{2}, t_{3}\right.$, etc. $)$. The different location of the parcels in the plume $\left(\left(x_{1}, y_{1}\right),\left(x_{2}, y_{2}\right)\right.$ etc. $)$, their distinctive local properties (Temperature $T_{1}, T_{2}$ or dilution ratio for instance) but also their position history lead to different aerosol size distribution and therefore to a non homogeneous distribution of the particle properties in the plume. 


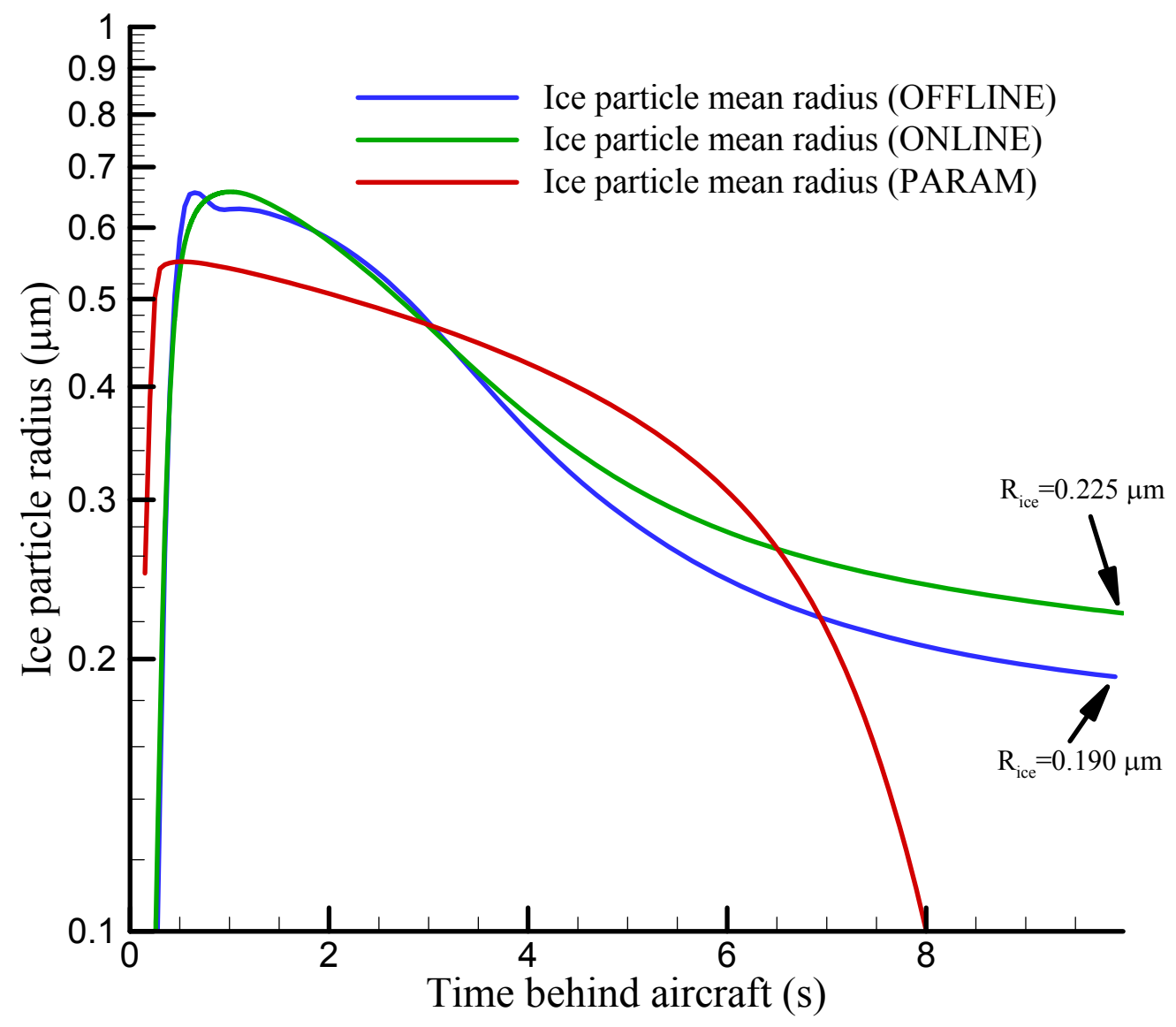

Figure 3: Time evolution of the ice particle mean radius in the plume of an Airbus A340 for three dilution calculation methods. 

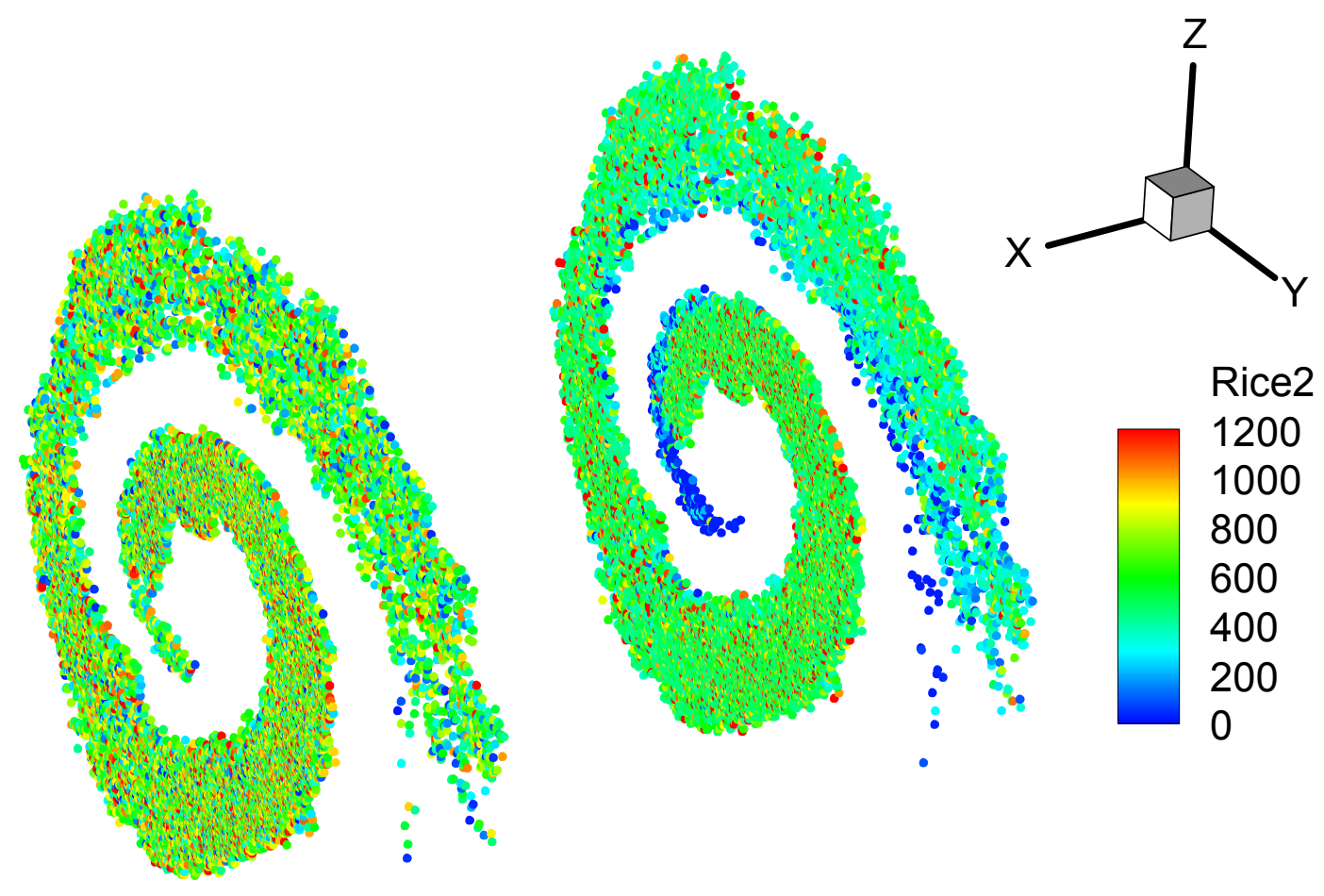

Figure 4: Slice of the plume at $t=1.8 \mathrm{~s}$ behind the aircraft. The ice particle radius is plotted for each numerical particle (i.e. for each of the 25000 positions). The results from FLUDILES (online) are shown on the left hand side and from ULP-ONE (offline) on the right hand side. 

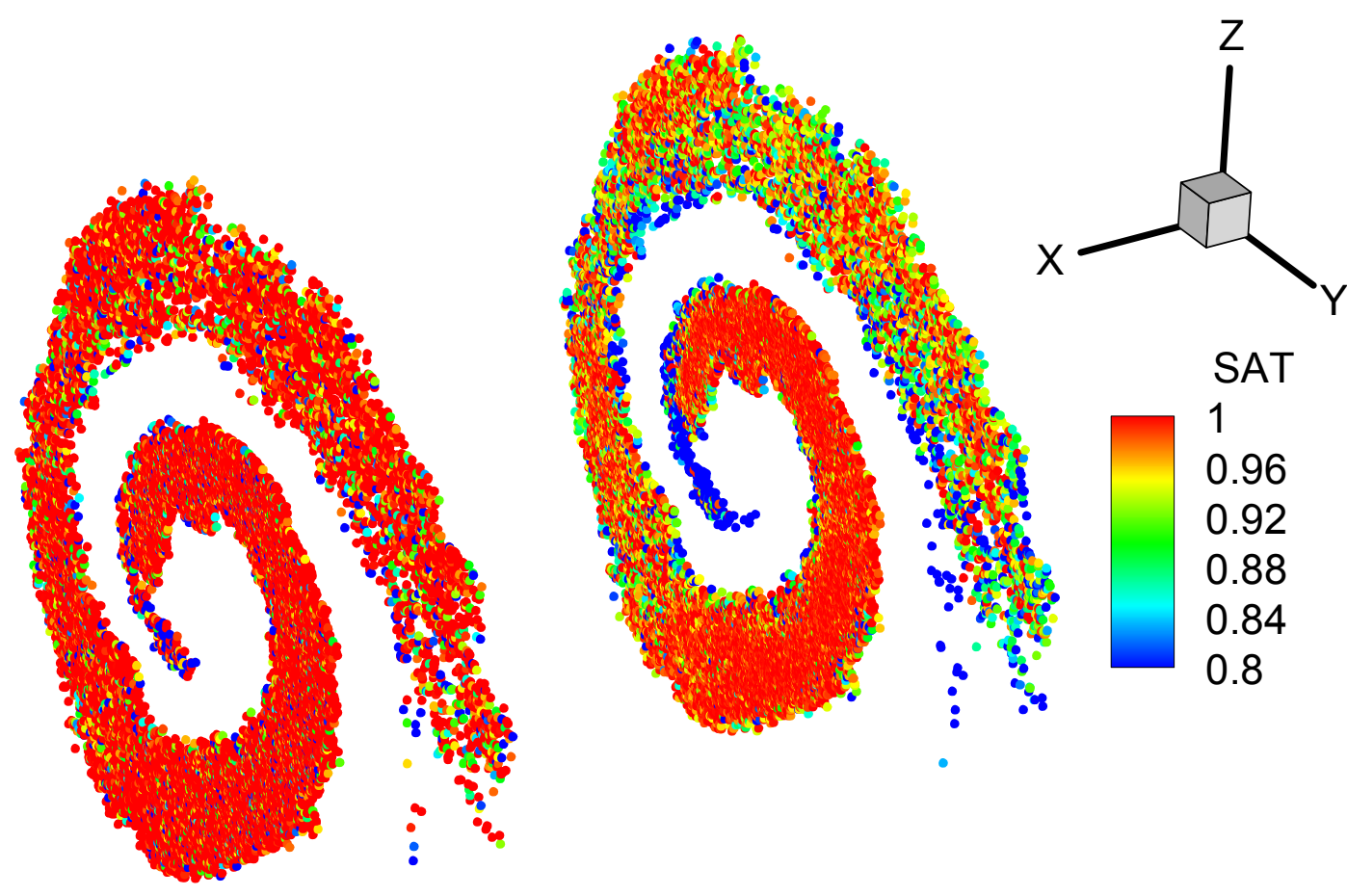

Figure 5: Slice of the plume at $t=1.8 \mathrm{~s}$ behind the aircraft. The water vapor saturation ratio is plotted for each numerical particle (i.e. for each of the 25000 positions). The results from FLUDILES (online) are shown on the left hand side and from ULP-ONE (offline) on the right hand side. 


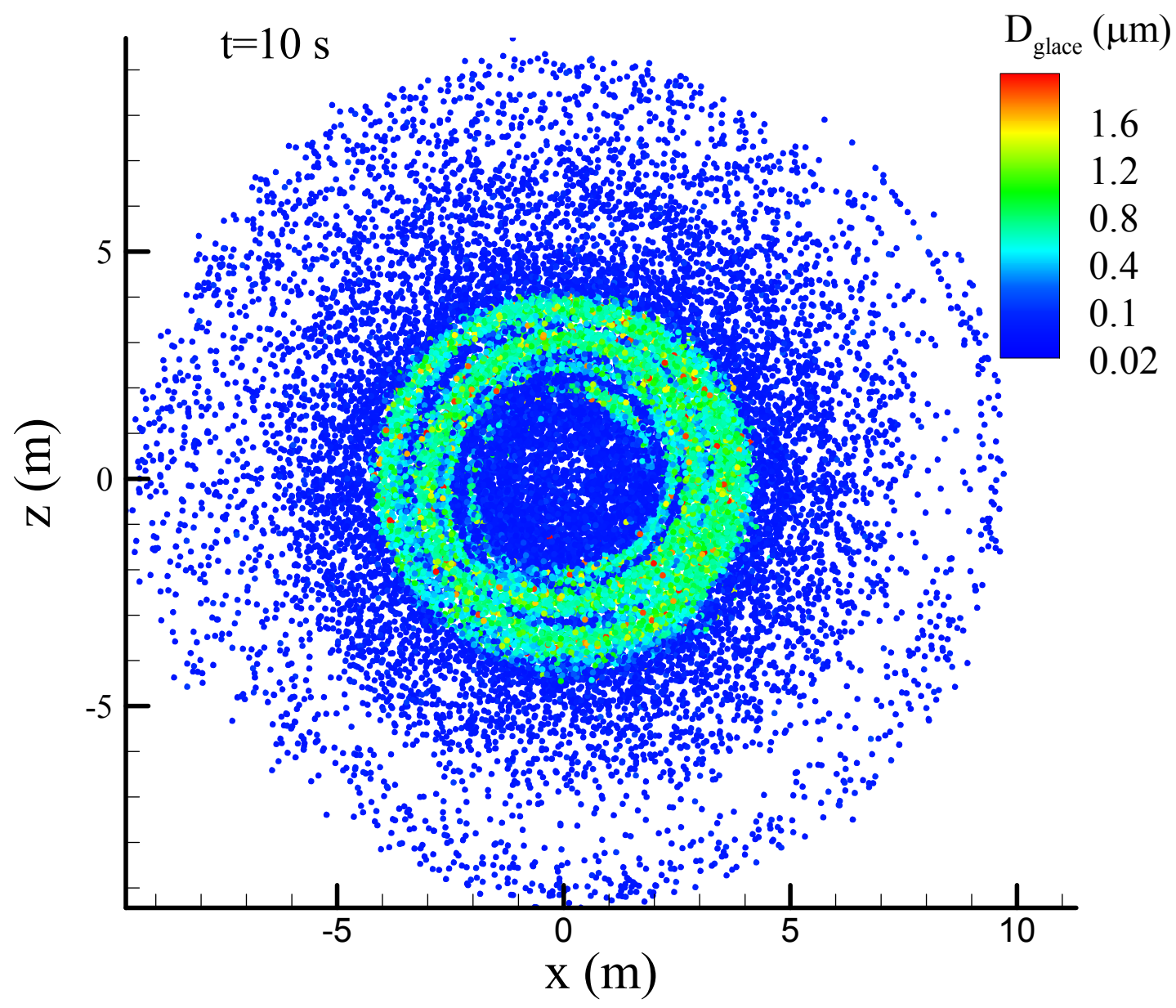

Figure 6: Ice particle mean diameter for each numerical particle position at $10 \mathrm{~s}$ behind the engine. 


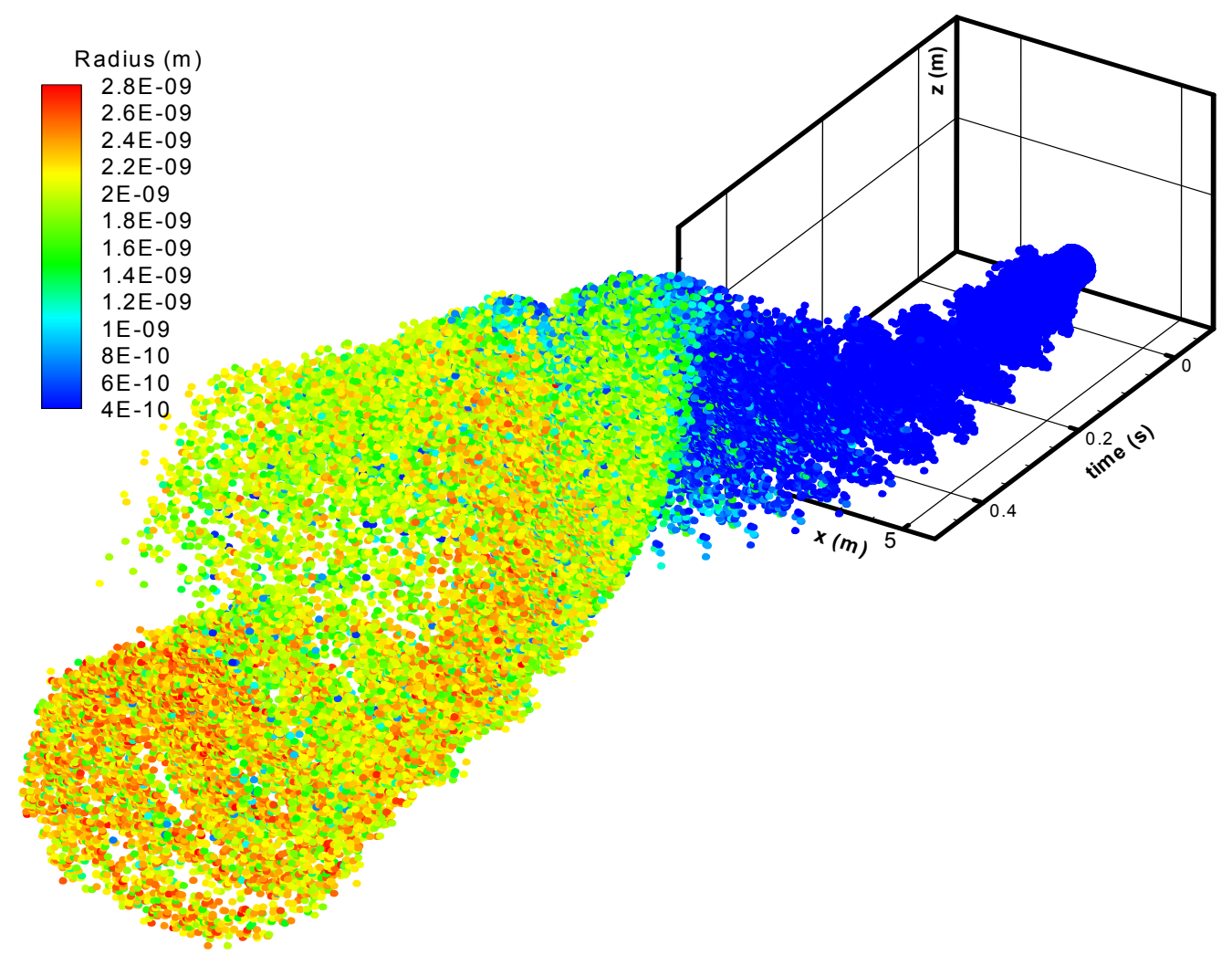

Figure 7: 2D slices of the plume represented at each time step. The value plotted is the volatile particle mean radius. 


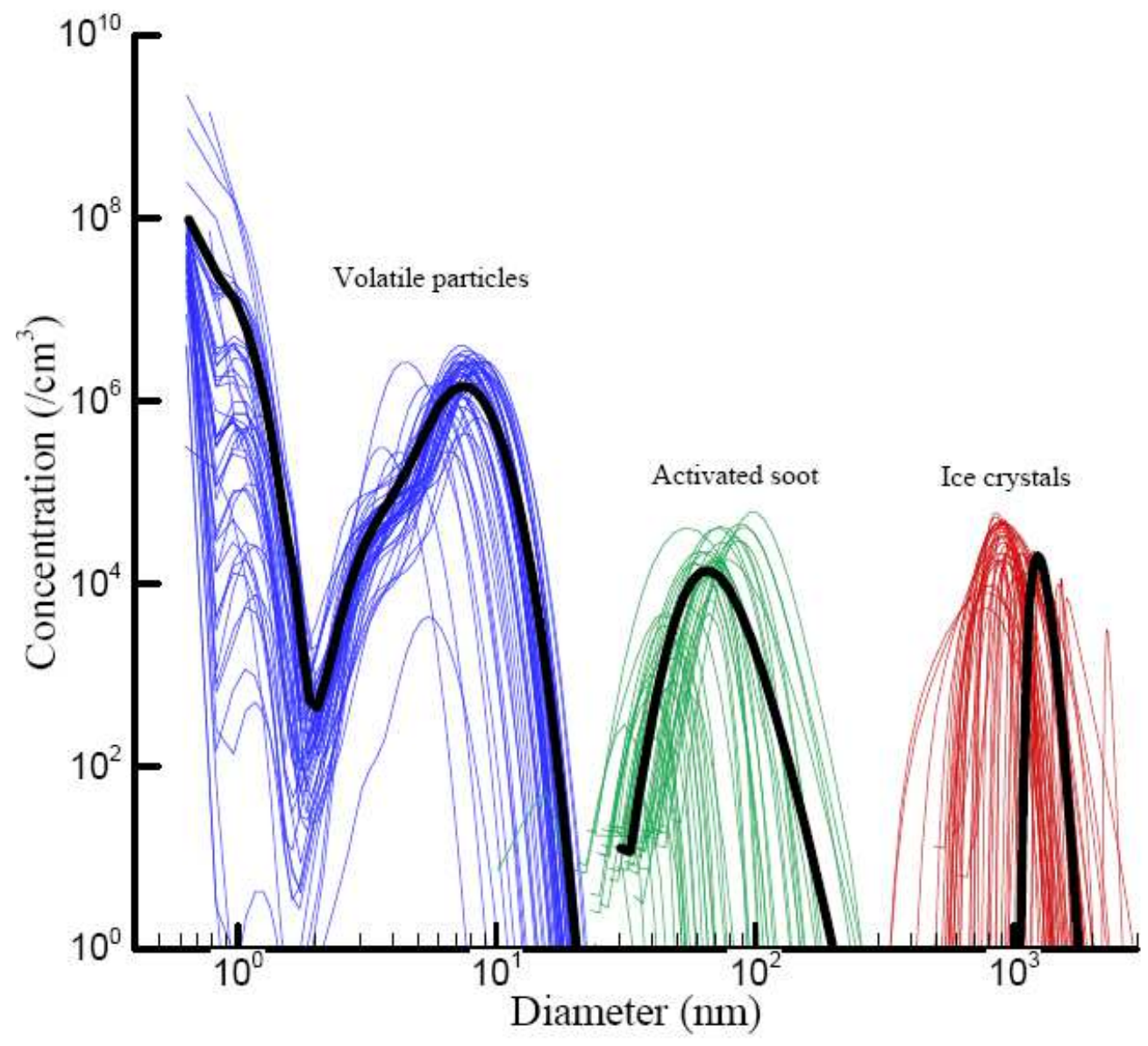

Figure 8: 50 randomly chosen size distribution of volatile particle, activated soot and contrail particles, $3 \mathrm{~s}$ behind the aircraft. The black line is the arithmetic average value. 